\title{
Influence of immune inflammation on androgen receptor expression in benign prostatic hyperplasia tissue
}

\author{
Zong-Lin $\mathrm{Wu}^{1}$, Ya Yuan ${ }^{2}$, He Geng ${ }^{1}$ and Shu-Jie $\mathrm{Xia}^{3}$
}

This study was designed to investigate the association between immune inflammation and androgen receptor (AR) expression in benign prostatic hyperplasia (BPH). We retrospectively analyzed 105 prostatectomy specimens. An immune inflammation score for each specimen was defined by combining three immunohistochemical markers (CD4, CD8 and CD20). The immunohistochemical markers were CD4 and CD8 for T lymphocytes, CD20 for B lymphocytes and AR antibody for the AR in BPH samples. Rates of CD4, CD8, CD20 and AR expression in BPH were 20 (19.0\%), 21 (20.0\%), 101 (96.2\%) and $48(45.7 \%)$, respectively. Total prostate volume (TPV) was higher in the immune inflammation group than in the non-immune inflammation group $(62.7 \mathrm{ml} v s .49 .2 \mathrm{ml}, t=2.482, P<0.05)$. Patients in the immune inflammation group had a higher serum prostate-specific antigen (PSA) than those in the non-inflammation group (7.5 $\mathrm{ng} \mathrm{ml}^{-1}$ vs. $\left.5.4 \mathrm{ng} \mathrm{ml}^{-1}, t=2.771, P<0.05\right)$. Specifically, the immune inflammation group showed a higher rate of AR expression than the non-inflammation group $\left(56.1 \%\right.$ vs. $\left.28.2 \%, \chi^{2}=7.665, P<0.05\right)$. Our study revealed a strong association between immune inflammation and TPV, serum PSA and AR expression in BPH tissue. Prostate hyperplasia caused by an immune inflammatory process may contribute to BPH progression over time. Therefore, the inflammatory response involved in BPH may be a prime therapeutic target.

Asian Journal of Andrology (2012) 14, 316-319; doi:10.1038/aja.2011.154; published online 12 December 2011

Keywords: androgen; androgen receptor; benign prostatic hyperplasia; immunohistochemistry; inflammation

\section{INTRODUCTION}

Benign prostatic hyperplasia (BPH) is one of the most common chronic conditions in aging men. ${ }^{1} \mathrm{BPH}$ is defined histologically as epithelial and stromal cell proliferation, which leads to prostate enlargement, low urinary tract symptoms and bladder outlet obstruction (BOO). ${ }^{2}$ Prevalence rates of BPH increase with age, with $50 \%$ of men over 50 years of age and $90 \%$ of men over 80 years having the condition. ${ }^{3,4}$ Like most chronic diseases, $\mathrm{BPH}$ is progressive; it evolves from cell proliferation to clinical $\mathrm{BPH}$ with low urinary tract symptoms over an extended period. BPH symptoms often have a significant impact on the quality of life( $\mathrm{QoL}$ ), and if untreated, BPH often causes BOO, which can lead to acute urinary retention (AUR) and can ultimately require surgery. ${ }^{5}$

To date, we have no precise knowledge of the cellular and molecular processes underlying the pathogenesis of $\mathrm{BPH}$. Although the influence of androgens and estrogens has been demonstrated, hormonal factors alone may not fully explain the pathogenesis of BPH. Experiments demonstrate that androgens, the estrogen-to-androgen ratio, immune inflammation and growth factors may play roles, either singly or in combination, in the etiology of BPH. ${ }^{6,7}$ The association of BPH with inflammation was first proposed based on the coexistence of hyperplastic nodules and chronic inflammation in BPH tissue. ${ }^{8}$ Prostatic inflammation, a common feature of the adult prostate, is associated with the development and progression of $\mathrm{BPH}$. Chronic immune inflammation is a highly common histological finding in the adult prostate. The prevalence of inflammation in $\mathrm{BPH}$ tissue ranges from $43 \%$ to $98 \% .^{9}$ The origin of inflammation in the prostate remains a subject of debate and probably has many causes. Immune inflammation correlates with total prostate volume (TPV), symptom progression, declining QoL, risk for AUR and the need for surgery. ${ }^{1}$

Although current evidence demonstrates that immune inflammation, androgens and the androgen receptor (AR) play roles in the pathogenesis of $\mathrm{BPH}$, it is not clear whether immune inflammation affects AR expression in $\mathrm{BPH}$ tissue. Our aim was to explore the association between immune inflammation and AR expression in patients with $\mathrm{BPH}$ by the use of immunohistochemistry (IHC).

\section{MATERIALS AND METHODS}

Benign prostatic hyperplasia samples

Patients in this report were selected from 457 patients with BOO due to BPH who underwent transurethral or suprapubic prostatectomy between January 2004 and December 2009. Written informed consent was obtained from each patient, and the study was approved by our local research and ethics committee. We obtained 105 random paraffin-embedded BPH tissue samples from 457 prostate samples. The mean age of the patients was 74.2 years (range: 56-91 years). Suprapubic prostatectomy was performed in 28 patients, and transurethral resection of the prostate was performed in 77 patients. All 105 patients had low urinary tract symptoms and BOO attributed to BPH. If the serum prostate-specific antigen (PSA) or digital rectal

${ }^{1}$ Department of Urology, Putuo District People's Hospital, Shanghai 200060, China; ${ }^{2}$ Department of Pathology, Putuo District People's Hospital, Shanghai 200060, China and ${ }^{3}$ Department of Urology, Shanghai First People's Hospital, Shanghai Jiao Tong University, Shanghai 200080, China Correspondence: Professor SJ Xia (xsjurologist@163.com)

Received: 13 July 2011; Revised: 7 August 2011; Accepted: 15 September 2011; Published online: 12 December 2011 
examination was abnormal, an ultrasound-guided biopsy would be performed before the operation to exclude prostate cancer. Thirtyfour patients had a history of AUR. Eleven patients had bladder stones due to BPH. The mean preoperative PSA was $6.7 \mathrm{ng} \mathrm{ml}^{-1}$ (range: $0.2-$ $36.0 \mathrm{ng} \mathrm{ml}{ }^{-1}$ ). The TPV was determined by transrectal ultrasonography. The mean TPV was $57.4 \mathrm{ml}$ (range: $16.0-230.0 \mathrm{ml}$ ).

\section{Immunohistochemistry}

Paraffin-embedded BPH tissue samples were cut into 3- to 5- $\mu$ m-thick sections using a microtome (Leica RM2235; Leica Biosystems Melbourne Pty Ltd, Melbourne, Australia) and placed onto charged glass slides (Leica Biosystems Melbourne Pty Ltd). Affix Bond Universal Slide Labels were attached to the specimen and control slides according to the manufacturer's instructions. Three lymphocyte markers were evaluated by IHC using the following monoclonal antibodies: CD4 (mouse monoclonal antibody, 4B12; antigen retrieval, EDTA-based buffer, pH 9; 1:200; Leica Biosystems Newcastle Ltd, Newcastle Upon Tyne, UK); CD8 (mouse monoclonal antibody, 4B11; antigen retrieval, EDTA-based buffer, $\mathrm{pH}$ 9; 1:200; Leica Biosystems); and CD20 (mouse monoclonal antibody, MJ1; antigen retrieval, citrate-based buffer, $\mathrm{pH}$ 6; 1:200; Leica Biosystems). AR expression was evaluated by IHC using a monoclonal antibody to label AR (mouse monoclonal antibody, 2F12; antigen retrieval, EDTAbased buffer, $\mathrm{pH}$ 9; 1:50; Leica Biosystems). After the slides were loaded into the tray and the reagents were loaded into the marker containers, the sections were processed on an automated instrument (Bond-Max system; Leica Biosystems Melbourne Pty Ltd) for immunostaining using the Bond detection system, which is based on a 3,3'-diaminobenzidine detection kit (Bond-Max system; Leica Biosystems Melbourne Pty Ltd).

Quality control was performed according to our laboratory's IHC rules from the CLIA Guidelines ${ }^{10}$ and the proposed NCCLS guidelines for IHC. Positive and negative controls were established according to the manufacturer's instructions.

CD4, CD8, CD20 and AR antibodies were used for the characterization of immune cells and AR in the BPH tissue specimens. Two independent observers, including one senior pathologist, applied IHC parameters separately. The observers scored the presence of lymphocytes and other IHC markers as absent, low or high $(0 / 1 / 2){ }^{8}$ If there was no lymphocyte infiltration, the inflammation score was zero; if lymphocyte infiltration was less than $50 \%$ of the slice area, the inflammation score was one; and if lymphocyte infiltration was greater than $50 \%$ of the slice area, the inflammation score was two.

\section{Statistical analysis}

All 105 prostate samples were divided into two groups based on the inflammation score of the BPH tissue. Samples were considered to be in the immune inflammation group if the inflammation score was greater than or equal to 2; all other patients were classified into the nonimmune inflammation group. The influence of immune inflammation and non-immune inflammation on AR expression was compared using the Chi-square test. All other data were reported as mean \pm s.d. An independent sample $t$-test was used to determine statistical significance. $P<0.05$ was considered significant. Commercially available software was used for statistical analyses (SPSS Inc., Chicago, IL, USA).

\section{RESULTS}

IHC analysis of CD4, CD8, CD20 and AR was performed on all 105 surgery-derived BPH specimens. As shown in Figure 1, T lymphocytes and B lymphocytes were associated with BPH immune inflammation.

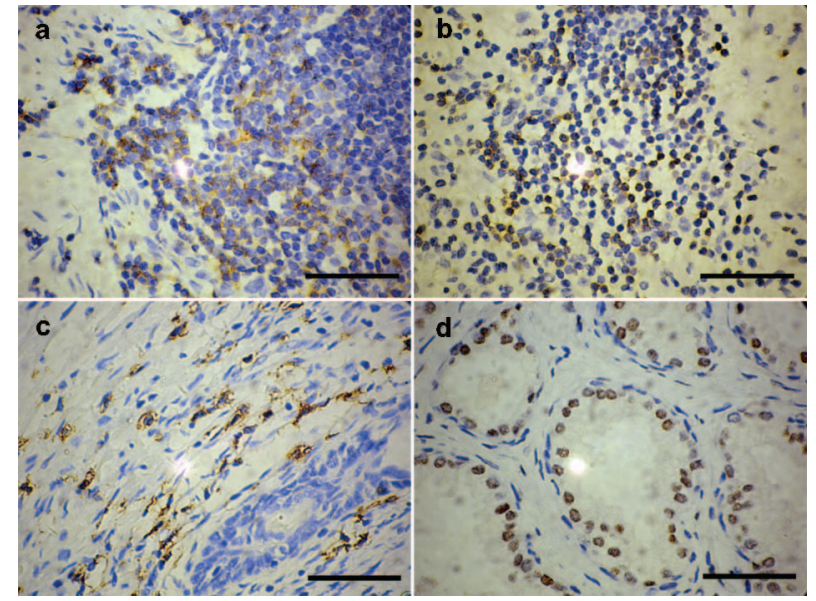

Figure 1 Immunostaining with $\mathrm{CD} 4, \mathrm{CD} 8, \mathrm{CD} 20$ and $\mathrm{AR}$ antibodies in $\mathrm{BPH}$ samples. (a) $\mathrm{CD}^{+}$; (b) $\mathrm{CD}^{+}$; (c) $\mathrm{CD}_{2} \mathrm{O}^{+}$; (d) $\mathrm{AR}^{+}$. Scale bars $=20 \mu \mathrm{m}$. AR, androgen receptor; $\mathrm{BPH}$, benign prostatic hyperplasia.

We observed an association between immune inflammation and AR expression in BPH. IHC analysis showed that CD4, CD8, CD20 and AR were expressed in 20 (19.0\%), 21 (20.0\%), 101 (96.2\%) and 48 (45.7\%) patients, respectively. The frequencies of IHC phenotypes were summarized in Table 1 . The $\mathrm{CD} 4^{-} \mathrm{CD} 8^{-} \mathrm{CD} 20^{+} \mathrm{AR}^{-}$phenotype and $\mathrm{CD} 4^{-} \mathrm{CD} 8^{-} \mathrm{CD} 20^{+} \mathrm{AR}^{+}$phenotype were expressed in $43(41.0 \%)$ and 27 (25.7\%), respectively, all above two phenotypes included CD20 expression.

The correlation of clinical parameters with immune inflammation and AR expression are shown in Table 2. There were six patients without recorded PSA values and 11 patients without TPV values. TPV was higher in the immune inflammation group than that in the non-immune inflammation group $(62.7 \mathrm{ml} v s .49 .2 \mathrm{ml}, t=2.482$, $P<0.05)$. Similarly, the level of PSA was associated with immune inflammation; mean PSA level in the immune inflammation group was higher than that in the non-inflammation group $\left(7.5 \mathrm{ng} \mathrm{ml}^{-1} v \mathrm{~s}\right.$. $\left.5.4 \mathrm{ng} \mathrm{ml}^{-1}, t=2.771, P<0.05\right)$. There was no significant difference between AR-expressing and AR-negative groups with respect to PSA, type of operation performed and history of AUR. However, TPV was significantly lower in the AR-expressing group than in the ARnegative group ( $51.6 \mathrm{ml}$ vs. $62.6 \mathrm{ml}, t=2.02, P<0.05)$. There was a significant difference in AR expression between the immune inflammation group and the non-immune inflammation group $\left(\chi^{2}=7.665\right.$, $P<0.05)$, as shown in Table 3 . The rate of AR expression in the

Table 1 Frequencies of immune inflammatory and AR phenotypes expressed by 105 patients

\begin{tabular}{lcccl}
\hline CD4 & CD8 & CD20 & $A R$ & $\mathrm{n}(\%)$ \\
\hline- & - & - & - & $3(2.9)$ \\
- & - & + & - & $43(41.0)$ \\
- & - & + & + & $27(25.7)$ \\
- & + & - & + & $1(1.0)$ \\
- & + & + & - & $5(4.8)$ \\
- & + & + & + & $6(5.7)$ \\
+ & - & + & - & $2(1.9)$ \\
+ & - & + & + & $9(8.6)$ \\
+ & + & + & - & $4(3.8)$ \\
+ & + & + & + & $5(4.8)$
\end{tabular}

Abbreviations: AR, androgen receptor; (-), absence of expression; $(+)$, presence of expression. 
Table 2 Comparison of clinical parameters between positive expression and negative expression of lymphocytes and AR antibodies

\begin{tabular}{|c|c|c|c|c|}
\hline & \multicolumn{2}{|c|}{ Immune inflammation ${ }^{a}$} & \multicolumn{2}{|c|}{$A R$} \\
\hline & + & - & + & - \\
\hline Age, $\bar{\chi} \pm$ s.d. (years) & $75.1 \pm 6.7(n=66)$ & $73.1 \pm 6.9(n=39)$ & $75.3 \pm 7.0(n=48)$ & $74.0 \pm 6.4(n=57)$ \\
\hline PSA, $\bar{\chi} \pm$ s.d. $\left(\mathrm{ng} \mathrm{ml}{ }^{-1}\right)$ & $7.5 \pm 3.8(n=60) *$ & $5.4 \pm 3.5(n=39)$ & $7.1 \pm 4.3(n=46)$ & $6.3 \pm 3.9(n=53)$ \\
\hline TPV, $\bar{\chi} \pm$ s.d. $(\mathrm{ml})$ & $62.7 \pm 26.6(n=60)^{*}$ & $49.2 \pm 22.9(n=34)$ & $51.6 \pm 27.2(n=41)^{\#}$ & $62.6 \pm 25.4(n=53)$ \\
\hline TURP (\%) & $62.3 \%(n=48)$ & $37.7 \%(n=29)$ & $50.6 \%(n=39)$ & $49.4 \%(n=38)$ \\
\hline
\end{tabular}

Abbreviations: AR, androgen receptor; AUR, acute urinary retention; $n$, valid data patient; PSA, prostate-specific antigen; TPV, total prostate volume; TURP, transurethral resection of prostate; $(-)$, absence of expression; $(+)$, presence of expression.

${ }^{a}$ Immune inflammation, if immune inflammation score was more than two.

$* P<0.05$, compared with non-immune inflammation group.

${ }^{\#} P<0.05$, compared with AR expression negative group.

immune inflammation group was higher than that in the noninflammation group (56.1\% vs. 28.2\%).

\section{DISCUSSION}

Androgens and the AR play an important role in the pathogenesis of $\mathrm{BPH}$. Testosterone is transformed into its metabolite dihydrotestosterone (DHT) by the enzymatic activity of $5 \alpha$-reductase type II in the prostate. Testosterone and DHT promote prostate stromal and epithelial cell growth and differentiation by two different mechanisms. One is the binding of the AR, which is expressed by epithelial and stromal cells, and the other is the induction of stromal synthesis of growth factors that act on epithelial and stromal compartments in a paracrine and an autocrine manner, respectively. ${ }^{11,12}$ The incidence and prevalence of $\mathrm{BPH}$ in men gradually increase with age. However, levels of circulating testosterone progressively decrease with age, suggesting that hormonal factors alone may not fully explain $\mathrm{BPH}$ pathogenesis. The development and hyperplasia of the prostate are regulated by other mechanisms besides sexual hormones, ${ }^{13-15}$ and immune inflammation appears to be a major player in the pathogenesis of BPH. ${ }^{6,16}$

With the recognition of the prostate's strategic role in keeping the reproductive tract sterile, it has become increasingly clear that this organ is an immunocompetent site and not an immunoprivileged site, as was previously thought. ${ }^{6}$ The prostate has fully active immunological functions and employs a broad spectrum of immune responses against foreign antigens. The normal prostate has only a small population of $\mathrm{T}$ cells, macrophages and $\mathrm{B}$ cells. CD8-positive $\mathrm{T}$ lymphocytes prevail over CD4-positive helper T cells. ${ }^{6}$

Studies by Theyer et al. ${ }^{17}$ and Kramer et al. ${ }^{18}$ have demonstrated that prostatic immune inflammatory cells consist of $70 \% \mathrm{~T}$ lymphocytes, 15\% B lymphocytes and 15\% macrophages, as well as mast cells.

Table 3 Association of immune inflammation with AR expression

\begin{tabular}{lllll}
\hline & & \multicolumn{2}{c}{$A R^{a}$} & \multirow{2}{*}{ Sum (n) } \\
\cline { 2 - 4 } & & \multicolumn{2}{c}{+} & \\
\hline Immune inflammation & - & $28(71.8 \%)$ & $11(28.2 \%)$ & 39 \\
\multirow{3}{*}{ Sum $(n)$} & + & $29(43.9 \%)$ & $37(56.1 \%)$ & 66 \\
& & 57 & 48 & 105 \\
\hline
\end{tabular}

Abbreviations: AR, androgen receptor; (-), absence of expression; (+), presence of expression.

${ }^{a}$ The rate of AR expression in the immune inflammation group was higher than in the non-inflammation group ( $56.1 \%$ vs. $\left.28.2 \%, \chi^{2}=7.665, P<0.05\right)$.
With aging, T-lymphocyte infiltration increases. At the same time, these researchers found that the ratio between CD8 cytotoxic/suppressor and CD4 helper T lymphocytes was reversed compared with the normal prostate, defined as the prostate in 18- to 25-year-old individuals and with a volume of $\leqslant 20 \mathrm{ml}$. In BPH tissue, immune inflammatory cells were organized into nodular infiltrates. Based on their anatomic localization within the prostate gland, immune inflammatory cells infiltration can be classified as glandular, periglandular and stromal. ${ }^{17}$

In our study, $96.2 \%$ of patients had significant B-lymphocyte infiltration. B lymphocytes were more numerous than other immune inflammatory cells and represented the major portion of the immune inflammatory infiltration observed in the prostate gland. Only $20 \%$ of patients had T-lymphocyte infiltration. Periglandular and stromal immune inflammatory infiltration were observed in our studies. Because of the unusual results, we performed the IHC twice. B lymphocytes are antigen-presenting cells that play a role in the first step of the immune system's inflammatory response. B lymphocytes may be stimulated directly by infectious agents or by prostatic epithelial cells. Several studies have demonstrated the presence of a heterogeneous set of bacterial and viral strains in BPH tissues. ${ }^{15} \mathrm{CD} 20$ antibodies to B lymphocytes were observed by IHC in almost all patients in our study, suggesting that bacterial or viral infection may be involved in the pathogenesis and progression of $\mathrm{BPH}$.

Experimental and clinical study data suggest that immune inflammation may stimulate prostate growth. Evidence has been found to support this hypothesis, and a possible mechanism has been proposed. di Silverio et al. ${ }^{19}$ retrospectively reviewed 3942 surgical BPH specimens and found that $43 \%$ of cases showed inflammation. The distribution of inflammatory characteristics varied significantly according to prostate volume. Chronic inflammation tended to vary with the prostate volume, suggesting a simple relationship between inflammation and prostatic hyperplasia. ${ }^{19}$ Several studies on BPH have found a connection between inflammation, TPV and PSA. The Medical Therapy of Prostatic Symptoms Study demonstrated a link between inflammation and higher TPV $(41.3 \mathrm{ml}$ vs. $36.8 \mathrm{ml}$, $P=0.0002) .{ }^{5}$ According to the REDUCE study, ${ }^{20}$ inflammation was associated with higher TPV ( $46.5 \mathrm{ml}$ vs. $43.4 \mathrm{ml}, P<0.0001)$. Robert et al. ${ }^{12}$ found that patients with high-grade inflammation had higher TPV ( $77 \mathrm{ml} v s .62 \mathrm{ml})$ than patients with low-grade inflammation.

We observed similar results in our study. TPV and PSA were significantly higher in the immune inflammation group compared to the non-immune inflammation group $(62.7 \mathrm{ml}$ vs. $49.2 \mathrm{ml}, t=2.482$, 
$P<0.05 ; 7.5 \mathrm{ng} \mathrm{ml}^{-1}$ vs. $5.4 \mathrm{ng} \mathrm{ml}^{-1}, t=2.771, P<0.05$, respectively). However, there was no significant difference between the immune inflammation group and the non-immune inflammation group with respect to the type of surgery performed.

The role of androgens and AR in the pathogenesis and progression of BPH has also been researched. Xia et al. ${ }^{22}$ observed low AR levels in the proximal duct $(29.71 \% \pm 4.02 \%)$, from which BPH originates, and an even lower AR in BPH tissue $(3.91 \% \pm 1.08 \%)$, suggesting a role of apoptosis in the pathogenesis of BPH. The expression of AR isoforms also varied among different $\mathrm{BPH}$ samples. The differences in expression of AR isoforms in prostatic tissues may explain why individual patients have different responses to hormone therapy. ${ }^{23}$ Humans synthesize two potent natural androgens, testosterone and DHT. ${ }^{24}$ DHT, the major intracellular androgen in the prostate, plays a crucial role in prostate development and growth and in the pathogenesis of BPH, as evidenced by the study of $5 \alpha$-reductase type II-deficient subjects, $5 \alpha$ reductase knockout animals and specific $5 \alpha$-reductase inhibitors. ${ }^{24}$ Testosterone and DHT are brought into action either by the ligand binding of AR or by the induction of stromal synthesis of growth factors. ${ }^{25}$ Further investigation of the association between immune inflammatory mediators and AR expression will increase our understanding of the interactions of immune components with AR and may facilitate the development of more specific and effective agents for the treatment of BPH.

In our study, there was a significant difference in AR expression between the immune inflammation group and the non-immune inflammation group $\left(\chi^{2}=7.665, P<0.05\right)$. The rate of AR expression in the immune inflammation group was higher than in the noninflammation one ( $56.1 \%$ vs. $28.2 \%$ ). However, TPV was significantly lower in the AR-expressing group than in the AR-negative patients (51.6 ml vs. $62.6 \mathrm{ml}, t=2.02, P<0.05)$. According to Xia et al., ${ }^{22} \mathrm{AR}$ expression is low in BPH tissue. This may explain the finding that larger TPVs appear to be associated with lower AR expression.

Immune inflammation and androgen-AR interactions may play a role in the pathogenesis of BPH. Our study demonstrates that the rate of AR expression in the immune inflammation group was higher than in the non-inflammation one. However, the mechanisms of immune inflammation that affect AR expression are not clearly defined. Inflammatory cells secrete $\mathrm{IL}-6,{ }^{12}$ which induces AR expression through the mitogen-activated protein kinase pathway. ${ }^{26}$ Further studies exploring these mechanisms will provide deeper insights into the immune inflammation-AR interaction in the prostate and spur the development of specific agents for the treatment of BPH.

In conclusion, the association between immune inflammation and AR expression was investigated in surgical $\mathrm{BPH}$ specimens. The results indicated a strong association between immune inflammation and TPV, PSA and AR expression in BPH tissue. Our findings indicated that the inflammatory process involved in $\mathrm{BPH}$ may be an important therapeutic target.

\section{AUTHOR CONTRIBUTIONS}

ZLW carried out the design of the study and drafted the manuscript. YY carried out the immunohistochemistry. HG participated in the design of the study and performed the statistical analysis. SJX conceived of the study, participated in its design and coordination, helped to draft the manuscript and have given final approval of the version to be published. All authors read and approved the final manuscript.

\section{COMPETING FINANCIAL INTERESTS}

There is no conflict of interest, financial or otherwise that could be perceived as prejudicing the impartiality of the research reported.

\section{ACKNOWLEDGMENTS}

We are grateful to Shu-Ping Jiang, Yao-Hua Sun, Hong-Bo Xu and Zhi-Cheng Dong for their technical support with the immunohistochemistry techniques, to Shu-Ping Jiang for obtaining the pathological images and picture processing, and to Shanghai Municipal Health Bureau for funding (No. 2009242).

1 Bushman W. Etiology, epidemiology, and natural history of benign prostatic hyperplasia. Urol Clin North Am 2009; 36: 403-15.

2 Roehrborn CG. Pathology of benign prostatic hyperplasia. Int J Impot Res 2008; 20 Suppl 3: S11-8.

3 Berry SJ, Coffey DS, Walsh PC, Ewing LL. The development of human benign prostatic hyperplasia with age. J Urol 1984; 132: 474-9.

4 Xia SJ, Xu XX, Teng JB, Xu CX, Tang XD. Characteristic pattern of human prostatic growth with age. Asian J Androl 2002; 4: 269-71.

5 McConnell JD, Roehrborn CG, Bautista OM, Andriole GL Jr, Dixon CM et al. The long-term effect of doxazosin, finasteride, and combination therapy on the clinical progression of benign prostatic hyperplasia. N Engl J Med 2003; 349 2387-98.

6 Kramer G, Mitteregger D, Marberger M. Is benign prostatic hyperplasia (BPH) an immune inflammatory disease? Eur Urol 2007; 51: 1202-16.

7 Zhou Y, Xiao XQ, Chen LF, Yang R, Shi JD et al. Proliferation and phenotypic changes of stromal cells in response to varying estrogen/androgen levels in castrated rats. Asian J Androl 2009; 11: 451-9.

8 Sciarra A, Mariotti G, Salciccia S, Gomez AA, Monti S et al. Prostate growth and inflammation. J Steroid Biochem Mol Biol 2008; 108: 254-60.

9 St Sauver JL, Jacobsen SJ. Inflammatory mechanisms associated with prostatic inflammation and lower urinary tract symptoms. Curr Prostate Rep 2008; 6 : 67-73.

10 Curren J. CLIA Compliance Handbook: The Essential Guide for the Clinical Laboratory. New York: Institute of Management \& Administration; 2007.

11 Penna G, Fibbi B, Amuchastegui S, Cossetti C, Aquilano F et al. Human benign prostatic hyperplasia stromal cells as inducers and targets of chronic immunomediated inflammation. J Immunol 2009; 182: 4056-64.

12 Fibbi B, Penna G, Morelli A, Adorini L, Maggi M. Chronic inflammation in the pathogenesis of benign prostatic hyperplasia. Int J Androl 2009; 32: 1-15.

13 Begley LA, Kasina S, MacDonald J, Macoska JA. The inflammatory microenvironment of the aging prostate facilitates cellular proliferation and hypertrophy. Cytokine 2008; 43: 194-9.

14 Lucia MS, Lambert JR. Growth factors in benign prostatic hyperplasia: basic science implications. Curr Urol Rep 2008; 9: 272-8.

15 Sciarra A, di Silverio F, Salciccia S, Autran Gomez AM, Gentilucci A et al. Inflammation and chronic prostatic diseases: evidence for a link? Eur Urol 2007. 52: $964-72$

16 Kramer G, Marberger M. Could inflammation be a key component in the progression of benign prostatic hyperplasia? Curr Opin Urol 2006; 16: 25-9.

17 Theyer G, Kramer G, Assmann I, Sherwood E, Preinfalk W et al. Phenotypic characterization of infiltrating leukocytes in benign prostatic hyperplasia. Lab Invest 1992; 66: 96-107.

18 Kramer G, Steiner GE, Handisurya A, Stix U, Haitel A et al. Increased expression of lymphocyte-derived cytokines in benign hyperplastic prostate tissue, identification of the producing cell types, and effect of differentially expressed cytokines on stromal cell proliferation. Prostate 2002; 52: 43-58.

19 di Silverio F, Gentile V, de Matteis A, Mariotti G, Giuseppe V et al. Distribution of inflammation, pre-malignant lesions, incidental carcinoma in histologically confirmed benign prostatic hyperplasia: a retrospective analysis. Eur Urol 2003; 43: $164-75$

20 Nickel JC, Roehrborn CG, O'Leary MP, Bostwick DG, Somerville MC et al. The relationship between prostate inflammation and lower urinary tract symptoms: examination of baseline data from the REDUCE trial. Eur Urol 2008; 54 $1379-84$.

21 Robert G, Descazeaud A, Nicolaiew N, Terry S, Sirab N et al. Inflammation in benign prostatic hyperplasia: a 282 patients' immunohistochemical analysis. Prostate 2009; 69: $1774-80$

22 Xia SJ, Xu CX, Tang XD, Wang WZ, Du DL. Apoptosis and hormonal milieu in ducta system of normal prostate and benign prostatic hyperplasia. Asian J Androl 2001; 3: 131-4.

23 Xia SJ, Hao GY, Tang XD. Androgen receptor isoforms in human and rat prostate. Asian $J$ Androl 2000; 2: 307-10.

24 Zhu YS, Imperato-McGinley JL. 5alpha-reductase isozymes and androgen actions in the prostate. Ann NY Acad Sci 2009; 1155: 43-56.

25 Li J, Al-Azzawi F. Mechanism of androgen receptor action. Maturitas 2009; 63 142-8.

26 Lin DL, Whitney MC, Yao Z, Keller ET. Interleukin-6 induces androgen responsiveness in prostate cancer cells through up-regulation of androgen receptor expression. Clin Cancer Res 2001; 7: 1773-81. 